\title{
Prospecção Tecnológica de Catalisadores Empregados em Reações de Transesterificação e Esterificação
}

\author{
Technological Prospection of Catalysts Employed in Transeterification \\ and Esterification Reactions
}

\author{
Nívea dos Santos Brainer ${ }^{1}$ \\ Wedja Timóteo Vieira ${ }^{1}$ \\ Rosana Correia Vieira ${ }^{1}$ \\ Rafael da Silva Oliveira de Holanda ${ }^{1}$ \\ Tatiane Luciano Baliano ${ }^{1}$ \\ João Inácio Soletti ${ }^{1}$ \\ Simoni Margareti Plentz Meneghetti ${ }^{1}$ \\ ${ }^{1}$ Universidade Federal de Alagoas, Maceió, AL, Brasil
}

\begin{abstract}
Resumo
O desenvolvimento de novos materiais é crescente ao longo das décadas, tendo como destaque os catalisadores, os quais exercem papel importante nos processos industriais atuando em reações como de transesterificação $e$ esterificação na produção de diversos compostos, a exemplo, os biocombustíveis. Neste trabalho realizou-se o levantamento prospectivo tecnológico de catalisadores utilizados em reações de transesterificação e esterificação. A execução das buscas de patentes e artigos ocorreu em diferentes bases de dados utilizando-se as palavraschave catalyst AND transesterification AND esterification. Essa temática tem atraído atenção no meio industrial $e$ acadêmico como observado no Scopus, com o número de publicações de 719 documentos, com os países líderes China e Índia; e no quesito número de patentes depositadas conforme o Patent Inspiration, de 216, destacando-se os Estados Unidos e Japão. O Brasil apresenta crescente interesse nesse cenário por meio do desenvolvimento e aperfeiçoamento de catalisadores.
\end{abstract}

Palavras-chave: Catalisador. Transesterificação. Esterificação.

\begin{abstract}
The development of new materials has been increasing over the decades, among which catalysts figure prominently playing an important role in industrial processes when it acts in reactions such as transesterification and esterification for the production of various compounds, such as biofuels. In this work a prospective technological survey of catalysts used in transesterification and esterification reactions was performed. Patent and article searching was performed on different databases using the keywords catalyst AND transesterification AND esterification. This issue has attracted attention in industry and academia as noted in Scopus with the number of publications of 719 documents, with China and India being the leading countries, and the number of patents filled of 216 according to Patent Inspiration, with United States and Japan having relevant positions. Brazil has a growing interest in this scenario through the development and improvement of catalysts.
\end{abstract}

Keywords: Catalyst. Transesterification. Esterification.

Área Tecnológica: Química. Catálise. 


\section{Introdução}

O aumento da demanda por fontes energéticas oriundas de combustíveis fósseis e a disponibilidade limitada destes recursos, assim como o aumento da conscientização ambiental, leva à busca por tecnologias mais limpas (ARANSIOLA et al., 2014). Diante disto, com o objetivo de minimizar os impactos ambientais e complementar as fontes energéticas existentes através de rotas sustentáveis, algumas alternativas estão sendo desenvolvidas como o uso de energias solar, eólica e biocombustíveis (YAN et al., 2015; ABBASZAADEH et al., 2012).

Nesse cenário, a oleoquímica é uma importante área de pesquisa e tecnologia em diversas instituições e indústrias por meio do desenvolvimento de novos produtos à base de gorduras e óleos para diferentes usos, em especial, para bioenergia e biocombustíveis (SALIMON; SALIH; YOUSIF, 2012).

Os triacilglicerídeos e ácidos graxos, presentes em óleos e gorduras de origem animal e vegetal apresentam um grande potencial reacional, decorrente da presença de diferentes funcionalidades em suas estruturas químicas. Assim, a partir destes compostos é possível obter diversos materiais com aplicações comerciais, porém, geralmente as transformações requerem a presença de um catalisador (SUAREZ et al., 2007). Portanto, catalisadores desempenham um papel fundamental nos processos industriais obtendo os produtos desejados em um menor tempo reacional, entre outros fatores.

Nas reações de transesterificação e esterificação, os catalisadores comumente utilizados podem ser de diferentes naturezas, como ácidos ou básicos de Brønsted, nas formas homogênea ou heterogênea. Na transesterificação de triacilglicerideos com álcool, na presença da base de Brønsted tais como hidróxidos e alcóxidos de sódio ou potássio, são obtidas altas conversões e seletividade para ésteres monoalquílicos. Entretanto, esses sistemas requerem matérias-primas de pureza elevada, evitando a ocorrência de reações indesejáveis como saponificação e hidrólise. Para esterificação são empregados ácidos de Brønsted, porém esses conduzem a taxas reacionais inferiores e são associados a problemas de corrosão nos reatores (SILVA et al., 2015; XIE; YANG; FAN, 2015).

Com o intuito de mitigar esses problemas, estudos mostraram que sistemas catalíticos exibindo sítios ácidos de Lewis apresentam resultados interessantes em esterificação, transesterificação e nessas reações simultâneas. Algumas vantagens desses sistemas são a minimização (i) da corrosão, quando se compara ao ácido sulfúrico empregado como catalisador na esterificação de ácidos graxos; e (ii) dos problemas de purificação na transesterificação, quando se utilizam óleos com alto teor de ácidos graxos livres (BRITO et al., 2012; SILVA et al., 2015).

Outro fator importante é a possibilidade de recuperação dos catalisadores, pois materiais com sítios ácidos de Lewis podem ser heterogêneos. A catálise heterogênea está despertando interesse de pesquisadores devido, principalmente, à fácil separação do meio reacional, o que proporciona a possibilidade de reutilização e regeneração do catalisador (EVANGELISTA et al., 2016).

Muitos estudos visando à investigação e desenvolvimento de catalisadores aplicados à transesterificação e esterificação estão sendo realizados (BANKOVIĆ-ILIĆ et al., 2017; SHARMA et al., 2018; JAMIL et al., 2018). Alguns exemplos desses sistemas catalíticos são àqueles a base de estanho(IV), amplamente utilizados nas reações de esterificação, transesterificação e poli- 
condensação para obtenção de polímeros e intermediários (LEE et al., 2003; SIDDARAMAIAH, 2004). Pesquisas demostraram potencial do uso desses catalisadores para obtenção de ésteres alquilícos (FERREIRA et al., 2007; SILVA et al., 2015; SERRA et al., 2011).

Nesse contexto, no campo da oleoquímica, os catalisadores ácidos são interessantes nas reações de transesterificação e esterificação para minimizar problemas operacionais e tornar processos mais eficientes e sustentáveis. Portanto, este trabalho visa realizar um levantamento prospectivo de sistemas catalíticos empregados nas reações de transesterificação e esterificação.

\section{Metodologia}

A prospecção dos dados foi baseada em artigos científicos, utilizando as bases SCOPUS (SCOPUS, 2018), WEB OF SCIENCE (WEB OF SCIENCE, 2018) e SCIENCE DIRECT (SCIENCE DIRECT, 2018). Para a investigação das patentes foram empregadas as plataformas nacionais e internacionais de acesso gratuito: Instituto Nacional da Propriedade Industrial (INPI, 2018), World Intellectual Property Organization (WIPO, 2018) e Patent Inspiration (PI, 2018).

As buscas dos dados foram realizadas por meio da combinação de palavras empregando operadores booleanos, a partir de 1952 a junho de 2018. Os termos pesquisados nas bases internacionais foram "catalyst transesterification", "catalyst esterification", "catalyst transesterification AND esterification", "catalyst oleochemistry" e "catalyst transesterification AND esterification AND oleochemistry". Os respectivos termos em português foram empregados na pesquisa em bases nacionais. A metodologia está ilustrada em forma de fluxograma na Figura 1.

Figura 1 - Fluxograma da metodologia empregada para pesquisas das patentes

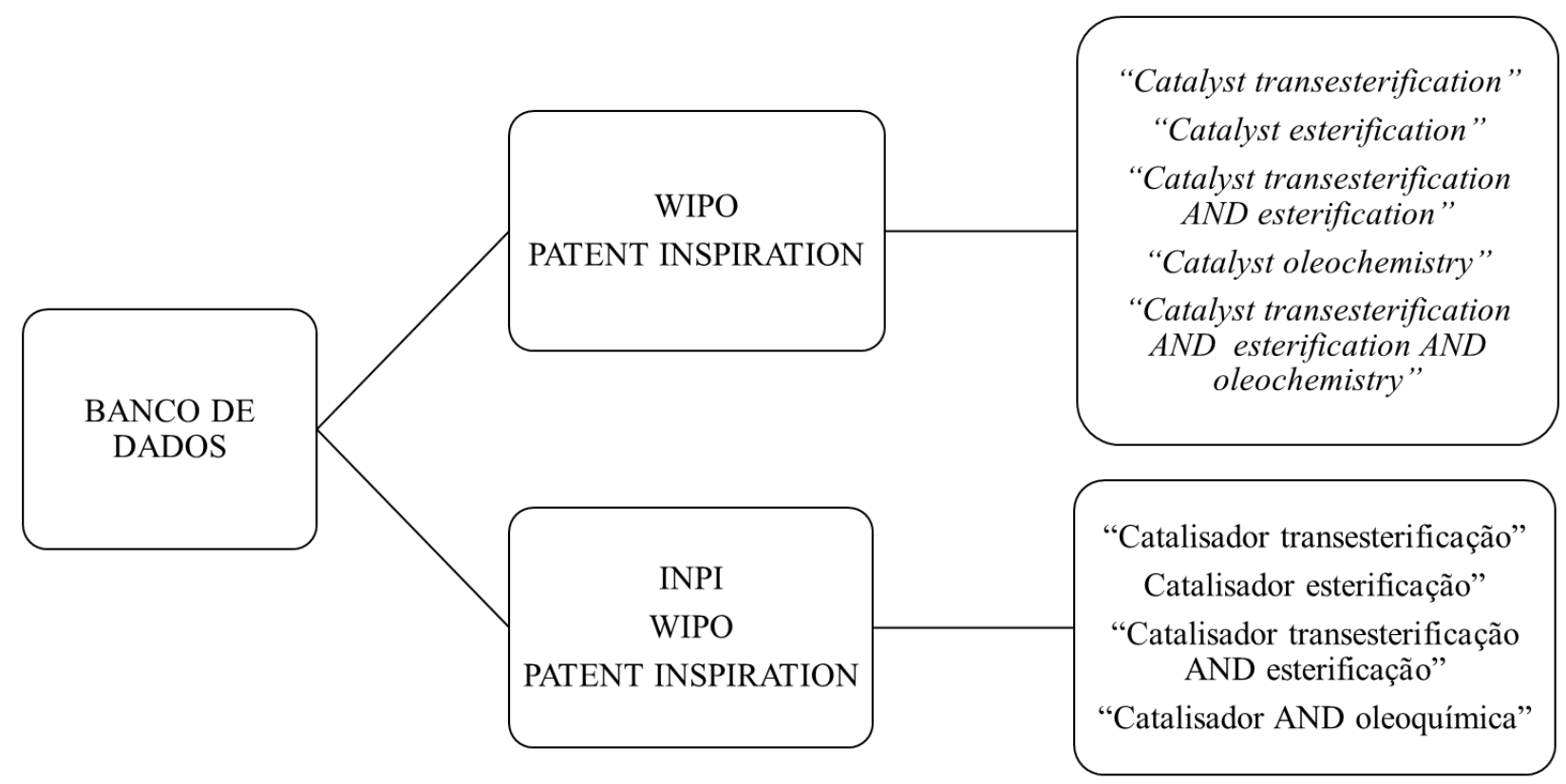

Fonte: Elaborada pelos autores deste artigo (2018)

Para o levantamento dos artigos científicos foram utilizadas as mesmas palavras-chave pela combinação dos campos título e/ou resumo, conforme a Figura 2. 
Figura 2 - Metodologia empregada para pesquisas dos artigos

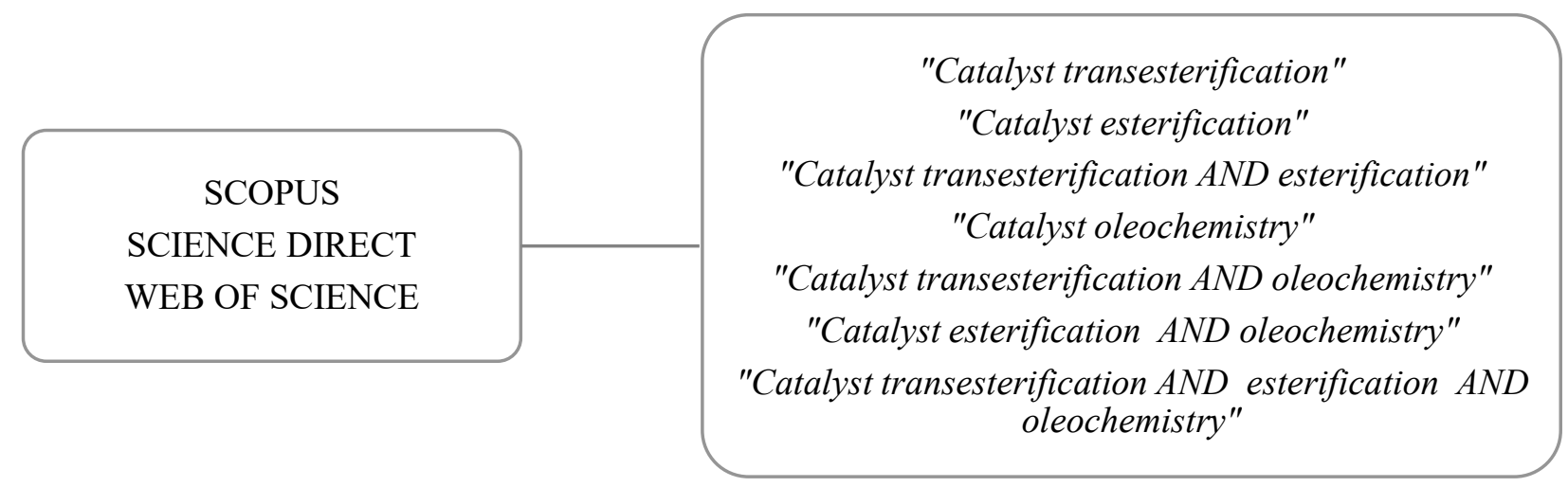

Fonte: Elaborada pelos autores deste artigo (2018)

\section{Resultados e Discussão}

Os resultados obtidos nas bases de dados, SCOPUS, SCIENCE DIRECT e WEB OF SCIENCE estão apresentados na Tabela 1 através do levantamento quantitativo de artigos publicados em periódicos científicos que abordam o tema conforme as palavras-chaves selecionadas. Dentre essas bases, a Scopus disponibiliza a maior quantidade de artigos publicados sobre o tema ao empregar todas as combinações de palavras-chave, 11.613, seguida das bases Web of Science com 5.570 e Science Direct com 4.631.

Tabela 1 - Número de artigos encontrados a partir das palavras-chave por base de periódicos.

\begin{tabular}{cccc} 
PALAVRAS-CHAVE & \multicolumn{3}{c}{ BASE DE DADOS } \\
& SCOPUS & $\begin{array}{c}\text { SCIENCE } \\
\text { DIRECT }\end{array}$ & $\begin{array}{c}\text { WEB OF } \\
\text { SCIENCE }\end{array}$ \\
$\begin{array}{ccc}\text { Catalyst AND Transesterication } \\
\text { Catalyst AND Esterification }\end{array}$ & 5.348 & 2.338 & 2.406 \\
$\begin{array}{c}\text { Catalyst AND Transesterification } \\
\text { AND Esterification }\end{array}$ & 719 & 1.904 & 2.365 \\
$\begin{array}{c}\text { Catalyst AND Oleochemistry } \\
\text { Catalyst AND Oleochemistry } \\
\text { AND Transesterification }\end{array}$ & 9 & 382 & 786 \\
$\begin{array}{c}\text { Catalyst AND Oleochemistry } \\
\text { AND Esterification }\end{array}$ & 3 & 4 & 9 \\
$\begin{array}{c}\text { Catalyst AND Oleochemistry AND } \\
\text { Transesterification AND Esterification }\end{array}$ & 1 & 1 & 2 \\
$\quad$ TOTAL & 11.613 & 4.631 & 1 \\
\hline
\end{tabular}

Fonte: Elaborada pelos autores deste artigo (2018)

Inicialmente, ao buscar os termos "catalyst" e "transesterification" como também "catalyst" e "esterification", obtêm-se números expressivos de artigos que fornecem dados a respeito da relevância científica desses materiais utilizados nessas reações. Ao realizar o refinamento na 
base de dados Scopus a partir de 1952 ao primeiro semestre de 2018, por meio da combinação das palavras Catalyst AND Transesterification AND Esterification, obteve-se 719 documentos publicados nessa temática. A Figura 3 apresenta os países com quantidade mais significativa de documentos, e pode-se observar que a China publicou o maior número, 134, seguida da Índia, Malásia e Estados Unidos com 116, 62 e 52, respectivamente. O Brasil é o quinto país com 48 documentos publicados. Esses dados indicam o crescimento das pesquisas científicas através da investigação e desenvolvimentos de novos materiais que possibilitam melhorar o desempenho das reações com maiores rendimentos, redução no tempo reacional, visando obter processos econômicos aliados ao meio ambiente (YADAV; UPADHYAY; SHARMA, 2018; PEDRO et al., 2018; HOSSAIN et al., 2018).

Figura 3 - Número de artigos por país
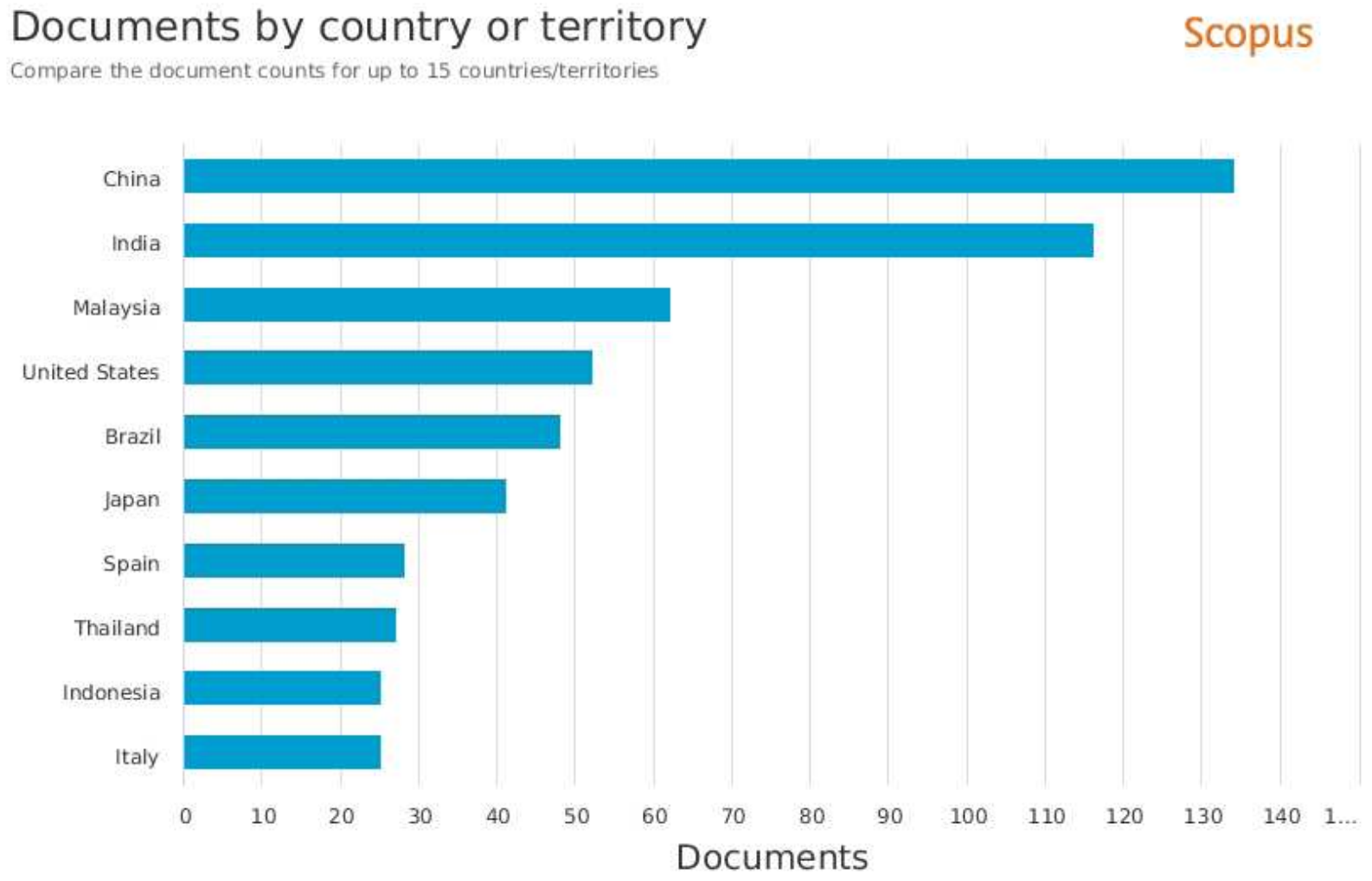

Copyright o 2018 Elsevier B.V. All rights reserved. Scopus iti is a registered trademark of Elsevler B.V.

Fonte: Elaborada pelos autores deste artigo (2018)

Dos 48 artigos publicados no Brasil, cerca de 17\% foram em 2017, sendo o maior número de documentos por ano, considerando-se o intervalo pesquisado. Dentre eles, alguns trabalhos realizam a investigação de catalisadores a base de $\mathrm{Sn}(\mathrm{IV})$ para a metanólise do óleo de soja e a esterificação do ácido oleico com metanol, os quais exibem elevadas atividades, sendo estas maiores do que os catalisadores inorgânicos clássicos. Além disso, esses sistemas proporcionam uma redução nas etapas de neutralização e purificação dos produtos, aspectos relevantes que contribuem para a diminuição dos impactos ambientais e dos custos do processo (NUNES et al., 2017; SILVA et al., 2015; BRITO et al., 2012). Assim, ao longo dos anos é expressivo o desenvolvimento de calisadores empregados nas reações de esterificação e transesterificação, pois proporciona condições mais favoráveis e estão alinhados aos príncipios da química verde. Reações catalíticas são superiores às reações estequiométricas em que a atuação dos catalisado- 
res pode ser altamente seletiva e efetiva em transformações complexas e difíceis (LENARDÃO et al., 2003), Figura 4.

Figura 4 - Número de artigos publicados no Brasil

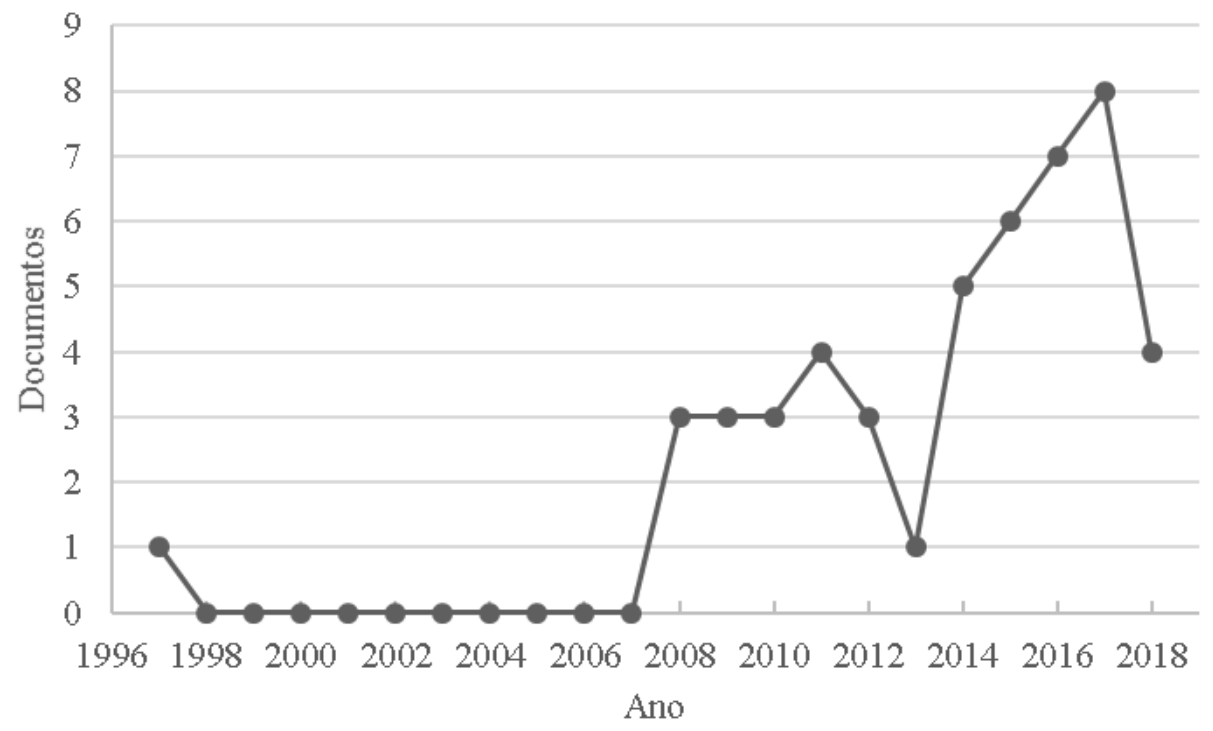

Fonte: Elaborada pelos autores deste artigo (2018)

As principais instituições que publicaram artigos estão apresentadas na Figura 5 , sendo a maioria universidades, com destaque a Academia de Ciência Chinesa com 21 artigos, Universidade da Malásia, 20, e Ministério da Educação da China com 19. No Brasil, a Universidade de Brasília publicou 8 artigos. Esses valores representam a atuação das instituições de ensino $e$ pesquisa no desenvolvimento de materiais aplicados às reações de esterificação e transesterificação e a relevância de incentivar e investir para a continuidade desses estudos.

Figura 5 - Número de documentos por afiliação

Documents by affiliation

compare the document counts for up to 15 affiliations

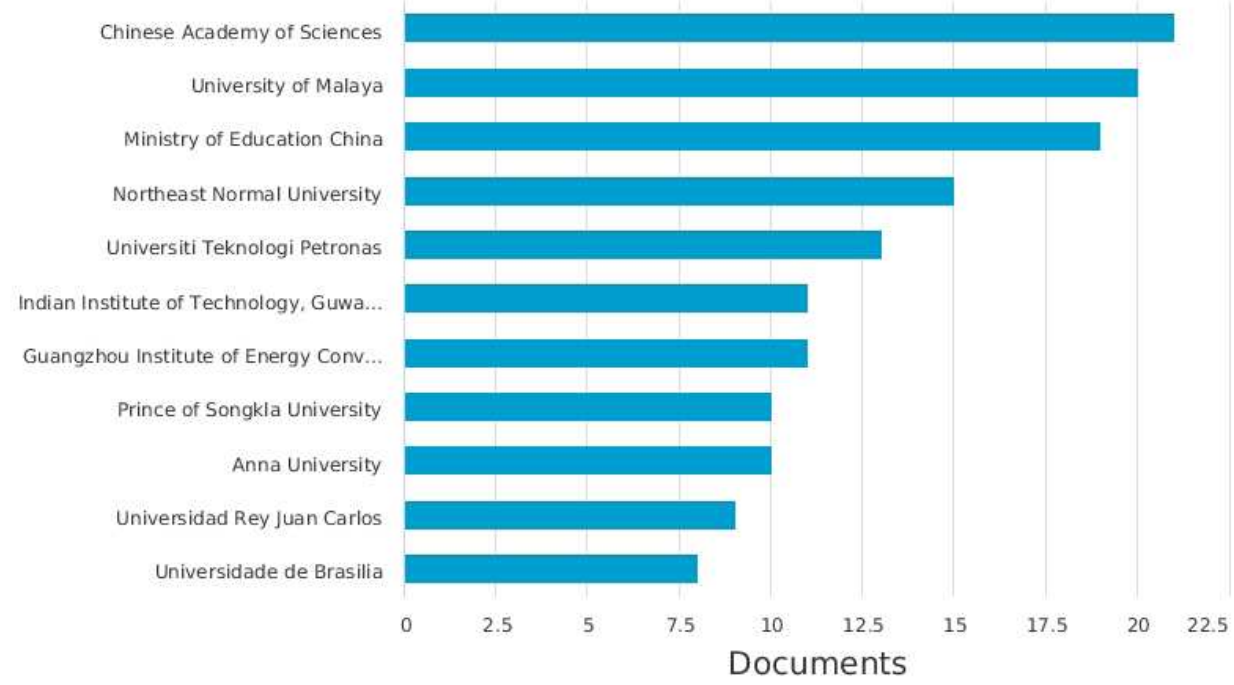

Copyright \& 2018 Elsevier B.V. All rights reserved. Scopusio is a registered trademark of Elsevier B.V

Fonte: Elaborada pelos autores deste artigo (2018) 
A Figura 6 ilustra a quantidade de artigos publicados ao longo dos anos, observando-se que, a partir de 2004, esses valores aumentaram. No ano de 2015 foram publicados 90 artigos representando o maior número, tendo um declínio de cerca de $29 \%$ nos dois anos seguintes. No ano de 2018, no primeiro semestre, 55 artigos foram publicados, correspondendo a aproximadamente $61 \%$ da quantidade de publicações em 2015.

Figura 6 - Número de artigos por ano de publicação

\section{Documents by year}

\section{Scopus}

100

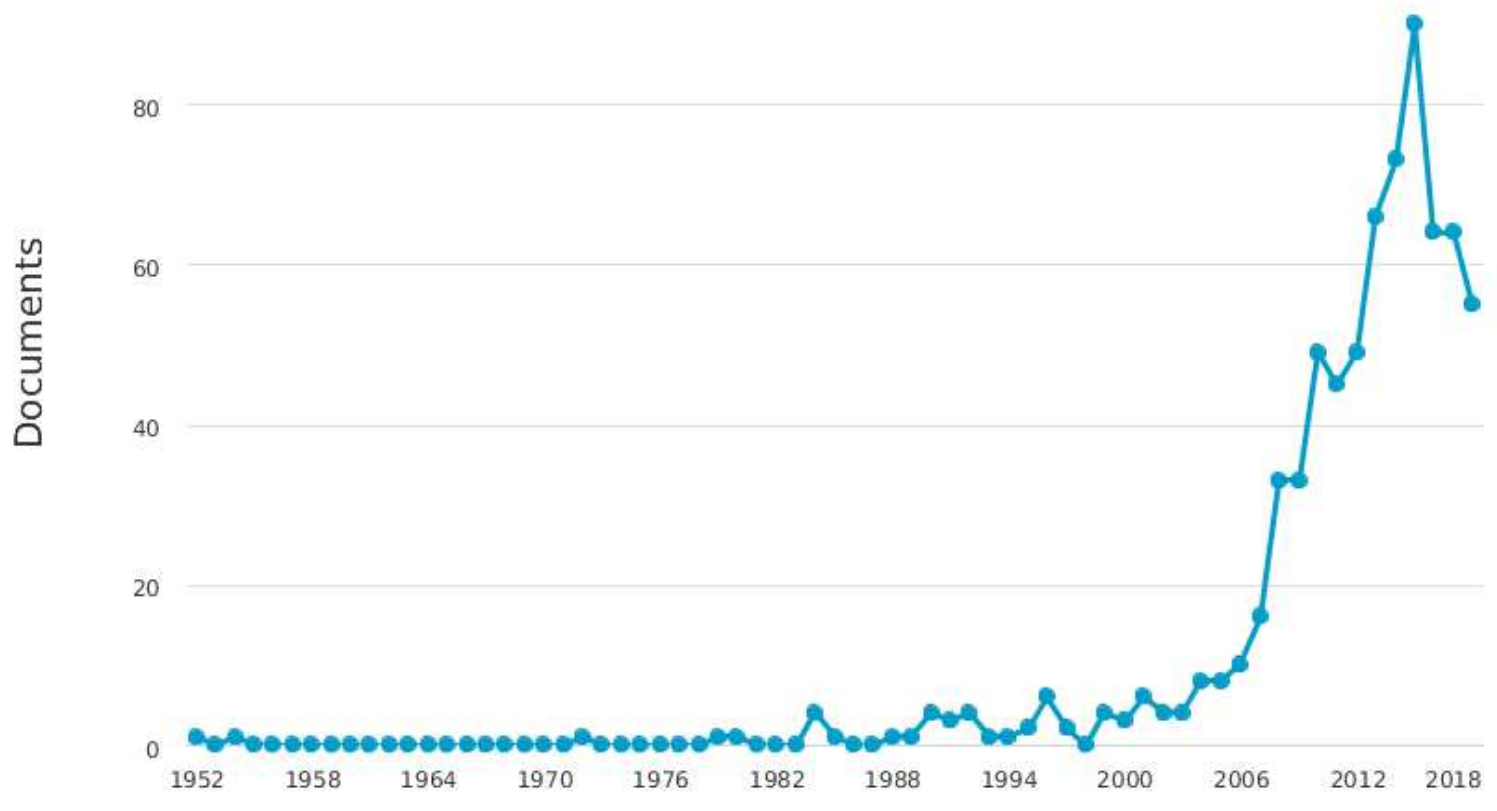

Fonte: Elaborada pelos autores deste artigo (2018)

A Figura 7 apresenta as áreas de publicações, sendo a área Engenharia Química, com 373 documentos, correspondente a 25,4\% do total, seguida da Química, 307 (20,9\%), Energia, 240 (16,3\%) e Ciência do meio ambiente, 135 (10,3\%). Na área de engenharia química, o biodiesel pode ser produzido a partir de fontes renováveis através de reações de transesterificação e esterificação na presença de catalisador. Trabalhos relatam o emprego de sistemas catalíticos heterogêneos, a exemplo do óxido de grafeno e materiais MCM-41 modificado, compostos que podem ser recuperados e reutilizados. Nesse sentido, o emprego desses materiais proporciona processos otimizados, tendo em vista a sustentabilidade e a redução dos impactos ambientais resultantes da utilização de matérias-primas renováveis e a diminuição de subprodutos indesejáveis no final do processo (QUITAIN et al., 2018; PIROUZMAND; ANAKHATOON; GHASEMI, 2018). 
Figura 7 - Número de artigos em relação a área

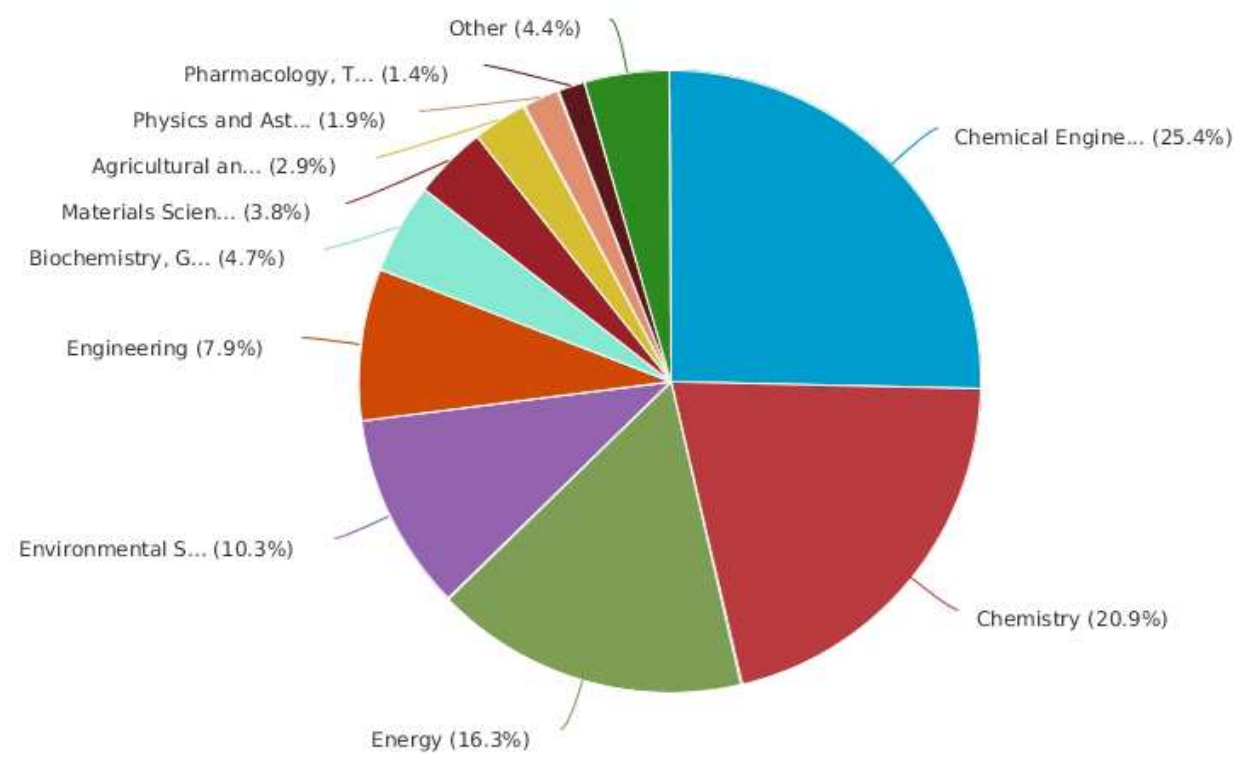

Copyright i 2018 Elsevier B.V. All rights reserved. Scopus is is a registered trademark of Elsevier B.V.

Fonte: Elaborada pelos autores deste artigo (2018)

Os resultados da pesquisa, pela combinação de palavras nas bases de patentes, se encontram na Tabela 2 . O refinamento realizado por meio da combinação de palavras foi compilado pelas bases, e a Patent Inspiration exibiu a maior quantidade de documentos patentários. A partir desta, os dados são representados graficamente (Figura 8).

Tabela 2 - Número de patentes encontradas nas bases de acordo com as palavras-chave

\begin{tabular}{lcccc} 
& PALAVRAS-CHAVE & \multicolumn{2}{c}{ BASE DE DADOS } \\
Titulo & Resumo & INPI & PI & WIPO \\
Catalyst & Transesterification & - & 690 & 663 \\
Catalyst & Esterification & - & 1.178 & 983 \\
Catalyst & Trasesterification AND Esterification & - & 216 & 228 \\
Catalyst & Oleochemistry & - & 0 & 0 \\
Catalyst & Transesterification AND Esterification & - & 0 & 0 \\
Catalisador & AND Oleochemistry & 29 & 45 & 21 \\
Catalisador & Transesterificação & 21 & 30 & 15 \\
Catalisador & Esterificação & 8 & 12 & 7 \\
Catalisador & Transesterificação AND Esterificação & 0 & 0 & 0 \\
Catalisador & Oleoquímica & 0 & 0 & 0 \\
Total & Transesterificação AND & $\mathbf{5 8}$ & $\mathbf{2 . 1 7 1}$ & $\mathbf{1 . 9 1 0}$ \\
\hline
\end{tabular}

Fonte: Patent Inspiration (2018) 
A Figura 8 apresenta os países que possuem expressivos números de patentes, e verifica-se que os Estados Unidos lideram com 98 patentes, correspondendo a $45 \%$ do total, seguido do Japão, 55, e Alemanha, 17. Isto demonstra o desenvolvimento realizado por esses países no emprego de catalisadores nas reações de transesterificação e esterificação para obtenção de diferentes produtos, a exemplo na área da oleoquímica, o biodiesel. Nos Estados Unidos, o catalisador nanoparticulado, formado pela mistura de óxido de zinco e óxido de lantânio, e o método utilizado nas reações foram patenteados. Esse catalisador heterogêneo atua simultaneamente nas reações de esterificação de ácidos graxos livres e transesterificação de triglicerídeos, possibilitando o emprego de maior diversidade de matérias-primas, pois não requer pureza desses precursores. Desta forma, óleo não refinado ou óleos de resíduos podem ser utilizados, não sendo necessárias as várias etapas de lavagem dos produtos, o que reduz significativamente água residual e a perda contínua do catalisador, diminuindo o custo de produção do produto final e os impactos causados ao ambiente (SHULI; STEVEN; SIMON, 2012).

Figura 8 - Número de patentes em relação ao país de origem
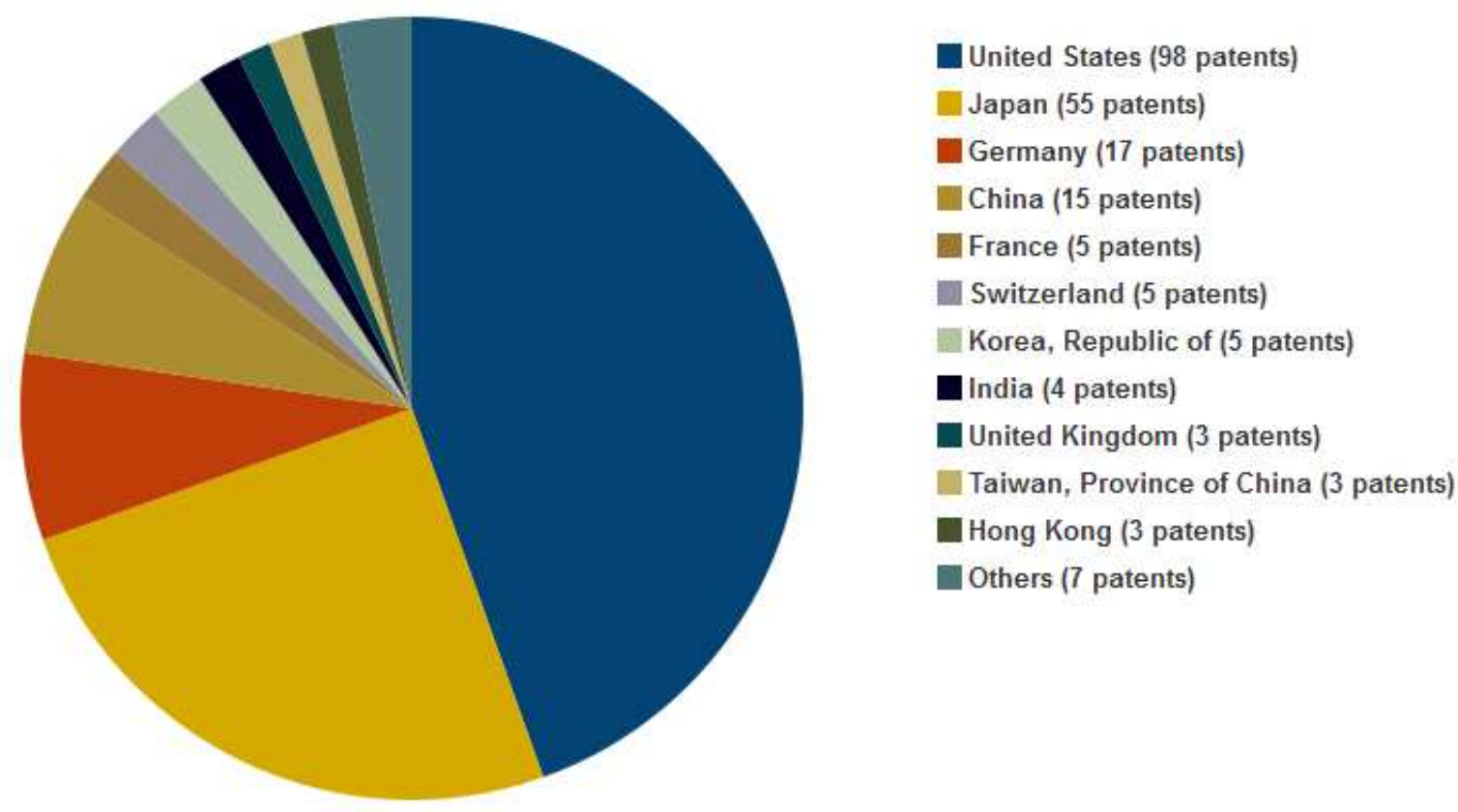

Fonte: Patent Inspiration (2018)

Quanto ao número de patentes por depositante, observa-se na Figura 9 uma dispersão nos resultados. A maioria dos depósitos é de grandes indústrias químicas, porém algumas universidades apresentam expressivos valores. A empresa Sakai Chemical Industry Co. possui uma família de patentes referente ao desenvolvimento de catalisador empregado em policondensação para obtenção de poliéster através de uma reação de transesterificação ou esterificação; enquanto a Universidade do Estado de Wayne possui patente de sistemas catalíticos de óxidos mistos metálicos suportados em zircônia utilizados nas reações de esterificação e transesterificação de óleo contendo ácidos graxos livres. 
Figura 9 - Número de patentes em relação ao depositante

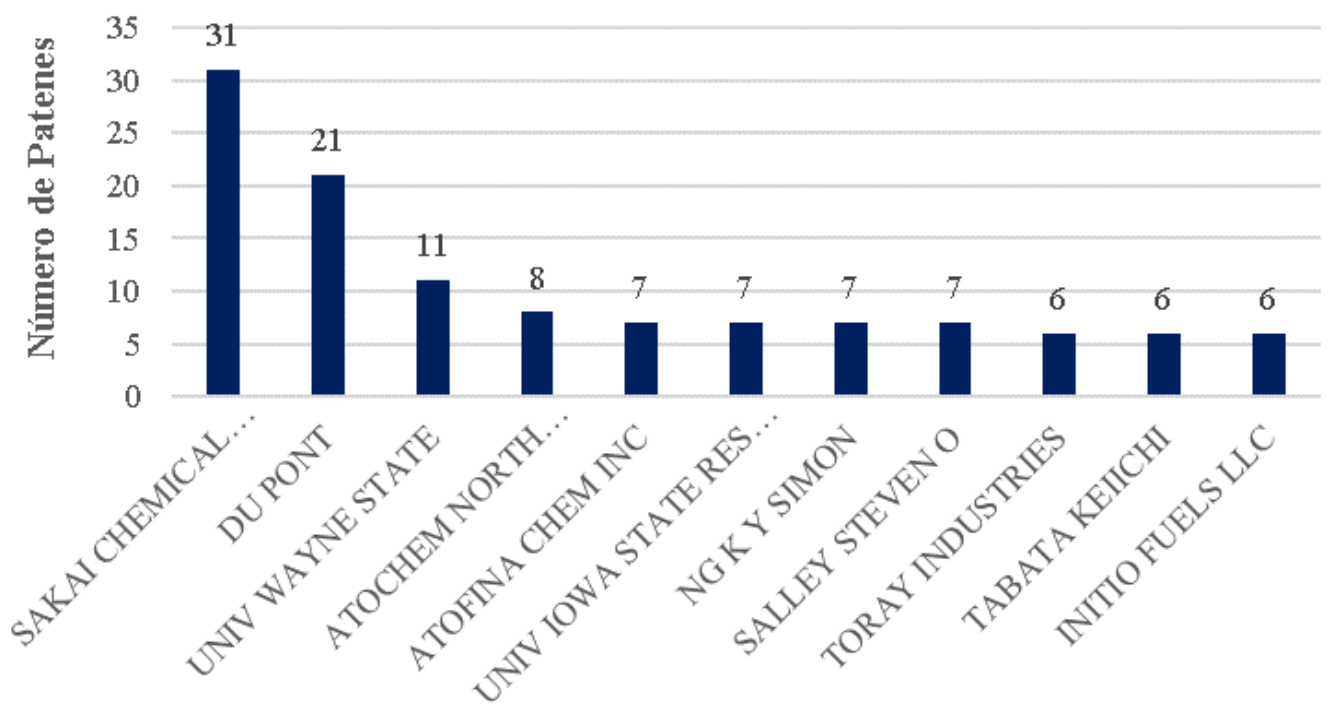

Depositantes

Fonte: Elaborada pelos autores deste artigo (2018)

Em relação à Classificação Internacional de Patentes (ICP), a Figura 10 reporta uma predominância na Classe C (Química; Metalurgia), com destaque na subclasse C08G63/00 com 88 patentes, referente a Compostos macromoleculares obtidos por reações formando uma ligação éster carboxílica de carbono na cadeia principal da macromolécula, e na subclasse C07C67/00, 68 patentes, Preparação de ésteres de ácidos carboxílicos. Na Classe B (Operações de processamento; Transporte) e a subclasse B01J31/00, 67 patentes, Catalisadores compreendendo hidretos, complexos de coordenação ou compostos orgânicos.

Figura 10 - Número de patentes em relação à classificação internacional de patentes

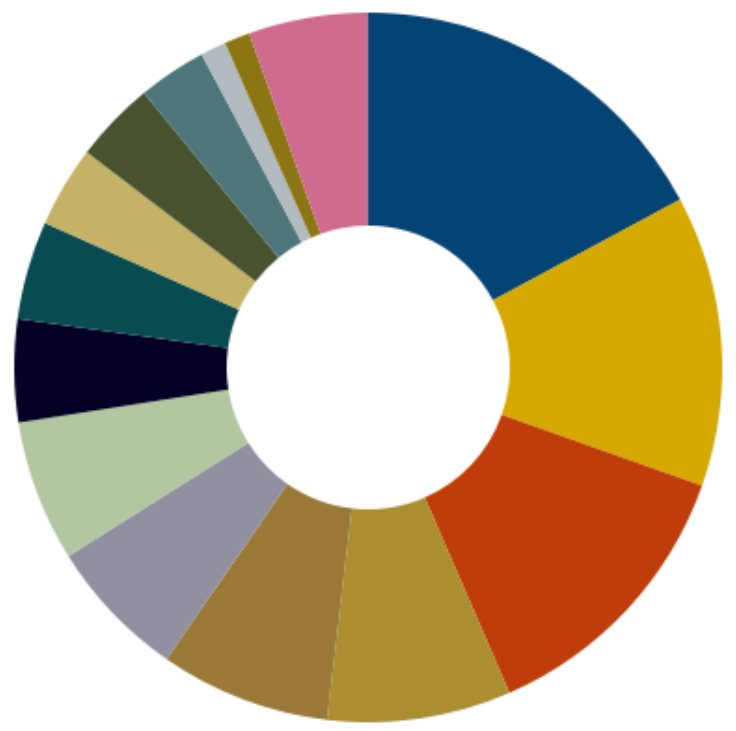

Fonte: Patent Inspiration (2018)
C08G63/00 (88 patents, 17\%) Macromolecular compounds obtained by reactions forming a carboxylic ester link in the main chain of the macromolecule

C 07 C67/00 (68 patents, 13\%) Preparation of carboxylic acid esters

B01J31/00 (67 patents, 13\%) Catalysts comprising hydrides

B01J23/00 (43 patents, 8\%) Catalysts comprising metals or metal oxides or hydroxides

a $07 \mathrm{C} 69 / 00$ (40 patents, $8 \%$ ) Esters of carboxylic acids

口 $11 \mathrm{C} 3 / 00$ (33 patents, 6\%) Fats

B01J21/00 (33 patents, 6\%) Catalysts comprising the elements

B01J27/00 (24 patents, 5\%) Catalysts comprising the elements or compounds of halogens

$\square$ C10L1/00 (23 patents, 4\%) Liquid carbonaceous fuels

C $07 F 7 / 00$ (19 patents, 4\%) Compounds containing elements of Groups 4 or 14 of the Periodic System

—C07B61/00 (19 patents, 4\%) Other general methods

B01J37/00 (16 patents, 3\%) Processes

C $08 \mathrm{~K} 5 / 00$ (6 patents, $1 \%$ ) Use of organic ingredients 
A Figura 11 reporta o número de patentes por ano de deposição, e observa-se que ocorreu um aumento expressivo a partir de 2000. Na última década foram publicadas 109 patentes, $e$ 88 a partir de 2010 a 2018, demostrando a importância de maiores investimentos das empresas e universidades para obterem processos mais eficientes com a presença de catalisadores.

Figura 11 - Número de patentes em relação ao ano de deposição

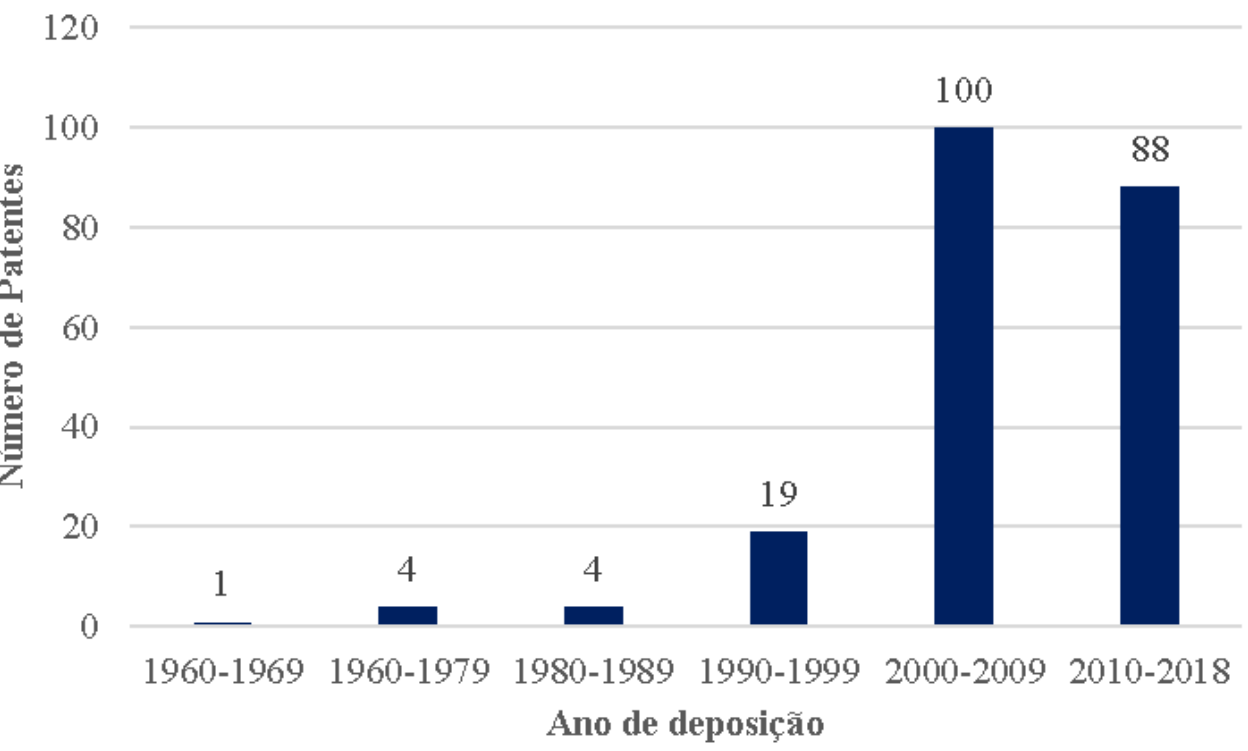

Fonte: Elaborada pelos autores deste artigo (2018)

Ao avaliar o Brasil nesse cenário, existem muitos trabalhos científicos sobre essa temática, principalmente no desenvolvimento de sistemas catalíticos bifuncionais, os quais atuam simultaneamente nas reações de esterificação e transesterificação, tais como: tricloreto de butilestanho, dicloreto de dibutilestanho, fostato de nióbio, zircônia sulfatada, inclusive através de processo catalítico enzimáticos empregando lipases comerciais (Novozym 435, Lipozyme RM IM e Lipozyme TL IM) (NUNES et al., 2017; ALESSIO et al., 2017; RAIA et al., 2017; PEDRO et al., 2018).

Em relação a patentes, o país não possui depósito conforme as pesquisas realizadas. Entretanto, ao avaliar pesquisas com as palavras-chaves "catalysts" e "transesterification", das 690 patentes depositadas (Tabela 2), cinco foram depositadas no Brasil, sendo três delas da mesma família de patentes que pertence ao campo de catalisadores para a transesterificação de triglicerídeos e descreve os métodos de síntese e formulação dos materiais, entre eles, o óxido de bário suportado em alumina e apatita. $\mathrm{E}$ as outras duas pertencentes à mesma família de patentes referem-se aos métodos utilizados para a transesterificação de óleos e gorduras através de diferentes catalisadores, como o pentóxido de nióbio. As patentes descritas foram depositadas pela empresa Petróleo Brasileiro S. A.

Ao analisar as patentes depositadas considerando-se as palavras-chaves "catalysts" e "esterification", apenas uma foi depositada no Brasil, pela Companhia Brasileira de Metalurgia e Mineração, que aborda sobre catalisadores heterogêneos ácidos como o óxido de nióbio hidratado e os processos de produção que podem ser aplicados em diferentes reações incluindo a esterificação.

Nesse contexto, o Brasil realiza diversas pesquisas de sistemas catalíticos aplicados na reação de esterificação e transesterificação como pode ser verificado nos trabalhos científicos publica- 
dos. Contudo, é importante a realização de estudos para a obtenção de materiais que sejam competitivos frente aos existes no mercado de modo que proporcionem processos eficientes, minimizando inconvenientes causados pelos existentes no mercado.

\section{Considerações Finais}

Através da prospecção tecnológica realizada, verificou-se que ao longo dos anos ocorreu um aumento significativo em desenvolvimento e investimento em novos materiais para obtenção de melhores condições reacionais. Assim, o número de patentes depositadas e artigos publicados tem crescido expressivamente. Os catalisadores atuam em numerosos processos sendo atrativos industrialmente nas reações como esterificação e transesterificação, então as indústrias lideram com o maior número de patentes depositadas, embora as universidades também desenvolvam pesquisas nessa temática. Em termos de trabalhos científicos abordando essa temática, países como China e Índia são destaques com maiores números de trabalhos publicados. Os Estados Unidos e Japão são líderes com maior número de patentes, demonstrando a importância desses compostos para a inovação tecnológica. Observa-se o aumento do número de documentos referentes ao desenvolvimento em pesquisas de catalisadores bifuncionais, os quais atuam simultaneamente nas reações, e possibilitam a produção de biocombustível, por exemplo, oriundos de diversas matérias-primas distintas, incluindo as com baixa qualidade e consideradas como resíduos. Além disso, contornam alguns problemas que estão presentes nos processos industriais.

Portanto, o levantamento prospectivo de sistemas catalíticos em reações de transesterificação e esterificação é importante, pois as indústrias químicas buscam por processos mais eficientes empregando materiais alternativos que possibilitem economia alinhada à sustentabilidade.

\section{Referências}

ABBASZAADEH, A.; GHOBADIAN, B.; OMIDKHAH, M. R.; NAJ, G. Current biodiesel production technologies: A comparative review. Energy Conversion and Management, [S.l.],v. 63, p. 138$148,2012$.

ALESSIO, C.; RIBEIRO, J. S.; CELANTE, D.; BRONDANI, L.; CASTILHO, F. Kinetics of methyl esters production with dimethyl carbonate over niobium phosphate. Energy Conversion and Management, [S.I.], v. 151, p. 670-680, 2017.

ARANSIONLA, E. F.; OJUMU, T. V.; OYEKOLA, O. O.; MADZIMBAMUTO, T. F; IKHU-OMOREGBE, D.I.O. A review of current technology for biodiesel production: State of the art. Biomass \&

Bioenergy, [S.l.], v. 61, p. 276-297, 2014.

BANKOVIĆ-ILIĆ, I. B.; MILADINOVIĆ, M.R.; STAMENKOVIĆ, O. S. ; VELJKOVIĆ, V. B. Application of nano $\mathrm{CaO}$-based catalysts in biodiesel synthesis. Renewable and Sustainable Energy Reviews, [S.l.], v. 72, p. 746-760, 2017.

BRITO, Y. C.; FERREIRA, D. A. C.; FRAGOSO, D. M. A.; MENDES, P. R.; OLIVEIRA, C. M. J.; MENEGHETTI, M. R.; MENEGHETTI, S. M. P. Simultaneous conversion of triacylglycerides and fatty acids into fatty acid methyl esters using organometallic tin(IV) compounds as catalysts. Applied Catalysis A: General, [S.l.], v. 443, p. 202-206, 2012. 
EPO - EUROPEAN PATENT OFFICE. Disponível em: https://worldwide.espacenet.com/ advancedSearch?locale $=$ en_EP. Acesso em: 21 jul. 2018.

EVANGELISTA, J. P. C.; GONDIM, A. D.; SOUZA, L. D.; ARAUJO, A. A. Aluminasupported potassium compounds as heterogeneous catalysts for biodiesel production: Areview. Renewable and Sustainable Energy Reviews, [S.I.], v. 59, p. 887-894, 2016.

FERREIRA, D. A. C.; MENEGHETTI, M. R.; MENEGHETTI, S. M. P. M.; WOLF, C. R. Methanolysis of soybean oil in the presence of tin(IV) complexes. Applied Catalysis A: General, [S.l.], v. 317, p. 58-61, 2007.

HOSSAIN, M. A.; IQBAL, M. A. M.; JULKAPLI, N. M.; KONG, P. S.; CHING, J. J.; LEE, H. V. Development of catalyst complexes for upgrading biomass into ester-based biolubricants for automotive applications: a review. Royal Society of Chemical Advances, [S.l.], v. 8, p. 5.5595.577, 2018.

INPI - INSTITUTO NACIONAL DA PROPRIEDADE INDUSTRIAL. Disponível em: https:/gru.inpi. gov.br/pePI/jsp/patentes/PatenteSearchAvancado.jsp. Acesso em: 21 jul. 2018.

JAMIL, F; AL-HAJ, L.; AL-MUHTASEB, A. H.; AL-HINAI, M. A.; BAAWAIN, M.; RASHID, U.; AHMAD, M. N. M. Current scenario of catalysts for biodiesel production: a critical review. Review in Chemical Engineering, [S.l.], v. 34, p. 267-297, 2018.

LEE, H.; KIM, S. J.; AHN, B. S.; LEE, W. K.; KIM, H. S. Role of sulfonic acids in the Sn-catalyzed transesterification of dimethyl carbonate with phenol. Catalysis Today, [S.I.], v. 87, p. 139-144, 2003.

LENARDÃO, E. J.; FREITAG, R. A.; DABDOUB, M. J.; BATISTA, A. C. F.; SILVEIRA, C. C. Green chemistry- The 12 principles of green chemistry and it insertion in the teac and research activities.

Química Nova, [S.l.], v. 26, p. 123-129, 2003.

NUNES, R. S.; ALTINO, F. M.; MENEGHETTI, M. R.; MENEGHETTI, S. M. P. New mechanistic approaches for fatty acid methyl ester production reaction in the presence of $\mathrm{Sn}(\mathrm{IV})$ catalysts.

Catalysis Today, v. 289, p. 121-126, 2017.

PEDRO, K. C. N. R.; PARREIRA, J. M.; CORREIA, I. N.; HENRIQUES, C. A.; LANGONE, M. A. P. Enzymatic biodiesel synthesis from acid oil using a lipase mixture. Química Nova, [S.l.], v. 41, p. 284-291, 2018.

PATENTINSPIRATION. [2018]. Disponível em: https://app.patentinspiration.com/\#report/ ac76C7f12E0F/filter. Acesso em: 21 jul. 2018.

PIROUZMAND, M.; ANAKHATOON, M. M.; GHASEMI, Z. One-step biodiesel production from waste cooking oils over metal incorporated MCM-41; positive effect of template. Fuel, [S.l.], v. 216, p. 296300, 2018.

QUITAIN, A. T.; SUMIGAWA, Y.; MISSION, E. G.; SASAKI, M.; ASSABUMRUNGRAT, S.; KIDA, T. G Graphene Oxide and Microwave Synergism for Efficient Esterification of Fatty Acids. Energy \& Fuel, [S.l.], v. 32, p. 3.599-3.607, 2018.

RAIA, R. Z.; DA SILVA, L. S.; MARCUCCI, S. M. P.; ARROYO, P. A. Biodiesel production from Jatropha curcas L. oil by simultaneous esterification and transesterification using sulphated zircônia.

Catalysis Today, [S.1.], v. 289, p. 105-114, 2017.

SALIMON, J.; SALIH, N.; YOUSIF, E. Industrial development and applications of plant oils and their 
biobased oleochemicals. Arabian Journal of Chemistry, [S.l.], v. 5, p. 135-145, 2012.

SCIENCE DIRECT. Disponível em: https://www.sciencedirect.com/search/advanced. Acesso em: 21 jul. 2018.

SCOPUS. Disponível em: https:/www-scopus.ez9.periodicos.capes.gov.br/search/form.uri?display= advanced\&origin $=$ searchbasic\&txGid=d614293cd7194d9c5032935634b02e2f. Acesso em: 21 jul. 2018.

SERRA, T. M.; MENDONÇA, D. R.; SILVA, J. P. V.; MENEGHETTI, M. R.; MENEGHETTI, S. M. P. Comparison of soybean oil and castor oil methanolysis in the presence of tin(IV) complexes. Fuel, [S.l.], v. 90, p. 2.203-2.206, 2011.

SHARMA, S.; SAXENA, V.; BARANWAL, A.; CHANDRA, P.; PANDEY, L. M. Engineered nanoporous materials mediated heterogneous catalyst and their implications in biodiesel production. Materials Science for Energy Technologies, [S.l.], v. 1, p. 11-21, 2018.

SHULI, Y.; STEVEN, S.; SIMON, N. K. Y. ZnO nanoparticle catalysts for use in transesterification and esterification reactions and method of making. Titular: University Wayve State, 2012.

SIDDARAMAIAH, M. B. Synthesis and characterization of polyurethane/ polybutyl methacrylate interpenetrating polymer networks. Journal of Materials Science, [S.I.], v. 39, p. 4.615-4.623, 2004.

SILVA, J. P. V.; BRITO, Y.C.; FRAGOSO, D. M. A.; MENDES, P. R.; BARBOSA, A. S. L.; BORTOLUZZI, J. H.; MENEGHETTI, M. R.; MENEGHETTI, S. M. P. Influence of different alkyl and carboxylate substituents on Sn(IV)

organometallic catalysts during fatty acid methyl ester production. Catalysis

Communications [S.I.], v. 58, p. 204-208, 2015.

SUAREZ, P. A. Z.; MENEGHETTI, S. M. P.; MENEGHETTI, M. R.; WOLF, C. R.;

Transformação de triacilglicerídeos em combustíveis, materiais poliméricos e insumos químicos: algumas aplicações da catálise na oleoquímica. Química Nova, [S.1.], v. 30, n. 3, p. 667-676, 2007.

WEB OF SCIENCE. Disponível em: http://apps-webofknowledge.ez9.periodicos.capes.gov.br/ WOS_AdvancedSearch_input.do?SID=5BGrLes5NHToCeKYOX9\&product=WOS\&search_ mode $=$ AdvancedSearch. Acesso em: 21 jul. 2018.

WIPO - World Intellectual Property Organization. Disponível em: https://patentscope.wipo.int/search/ pt/structuredSearch.jsf. Acesso em: 21 jul. 2018.

XIE, W.; YANG, X.; FAN, M. Novel solid base catalyst for biodiesel production:

Mesoporous SBA-15 silica immobilized with 1,3-dicyclohexyl-2-octylguanidine. Renewable Energy, [S.l.], v. 80, p. 230-237, 2015.

YADAY, M.; UPADHYAY, S.; SHARMA, Y. C. Process optimization, kinetics of production Jatropha curcus methyl ester, and its utilization in single cylinder diesel engine. Energy Conversion and Management, [S.I.], v. 160, p. 364-374, 2018.

YAN, K.; JARVIS, C.; GU, G.; YAN, Y. Production and catalytic transformation of levulinic acid: A platform for speciality chemicals and fuels. Renewable and Sustainable Energy Reviews, [S.1.], v. 51, p. 986-997, 2015. 


\section{Sobre os Autores}

\section{Nívea dos Santos Brainer}

E-mail: niveabrainer@gmail.com

Mestranda em Engenharia Química pela Universidade Federal de Alagoas.

Endereço profissional: Grupo de Catálise e Reatividade Química - GCAR. Universidade Federal de Alagoas UFAL, Campus A.C. Simões. Av. Lourival de Melo Mota s/n, Tabuleiro dos Martins. CEP: 57072-900.

\section{Wedja Timóteo Vieira}

E-mail: wedja.tvieira@gmail.com

Mestre em Engenharia Química pela Universidade Federal de Alagoas.

Endereço profissional: Laboratório de Sistemas de Separação e Otimização de Processos - LASSOP. Universidade Federal de Alagoas - UFAL, Campus A.C. Simões. Av. Lourival de Melo Mota s/n, Tabuleiro dos Martins. CEP: 57072-900.

\section{Rosana Correia Vieira}

E-mail: rosana1correia@hotmail.com

Mestranda em Engenharia Química pela Universidade Federal de Alagoas.

Endereço profissional: Laboratório de Tecnologias de Bebidas e Alimentos - LTBA. Universidade Federal de Alagoas - UFAL, Campus A.C. Simões. Av. Lourival de Melo Mota s/n, Tabuleiro dos Martins. CEP: 57072-900.

\section{Rafael da Silva Oliveira de Holanda}

E-mail: rafael.holanda@ctec.ufal.br

Mestrando em Engenharia Química pela Universidade Federal de Alagoas.

Endereço profissional: Laboratório de Eletroquímica Aplicada - LEAp. Universidade Federal de Alagoas - UFAL, Campus A.C. Simões. Av. Lourival de Melo Mota s/n, Tabuleiro dos Martins. CEP: 57072-900.

\section{Tatiane Luciano Baliano}

E-mail: tlb@qui.ufal.br

Doutora em Física Aplicada (Biomolecular) pela Universidade de São Paulo (USP) (2010).

Endereço profissional: Laboratório de Bioprocessos, Cristalografia e Modelagem Molecular - LaBioCriMM. Universidade Federal de Alagoas - UFAL, Campus A.C. Simões. Av. Lourival de Melo Mota s/n, Tabuleiro dos Martins. CEP: 57072-900.

\section{João Inácio Soletti}

E-mail: jisoletti@gmail.com

Pós-doutor na University of British Columbia, em Vancouver, Canadá. Doutor em Engenharia Química pela Universidade Federal do Rio de Janeiro/COPPE/PEQ (1997).

Endereço profissional: Laboratório de Sistemas de Separação e Otimização de Processos - LASSOP. Universidade Federal de Alagoas - UFAL, Campus A.C. Simões. Av. Lourival de Melo Mota s/n, Tabuleiro dos Martins. CEP: 57072-900.

\section{Simoni Margareti Plentz Meneghetti}

E-mail: simoni.plentz@gmail.com

Pós-Doutorada na Université Louis Pasteur Strasbourg, ULP, França. Doutora em Química e Físico Química de Materiais Macromoleculares (2000).

Endereço profissional: Grupo de Catálise e Reatividade Química - GCAR Universidade Federal de Alagoas UFAL, Campus A.C. Simões. Av. Lourival de Melo Mota s/n, Tabuleiro dos Martins. CEP: 57072-900. 